\title{
Academic Exploration
}

\section{Chinese Medicine, Which Targets Body's Response State, Is An Effective Way to Treat Epidemic Infectious Diseases}

\begin{abstract}
YUAN Bing
ABSTRACT In the fight against epidemic infectious diseases in the past 2,000 years, Chinese medicine (CM) has gradually developed a complete response system including diagnosis and treatment. The focal point of $\mathrm{CM}$ in the treatment of infectious diseases is the personalized response state to pathogen, which is a treatment method consistent with the personalized concept of precision medicine. Compared with the methods of directly killing or inactivating pathogens, which are used in modern medicine, $\mathrm{CM}$ is an effective treatment modality that has a wider range of points of action in the human body. The remarkable effects achieved while treating SARS in 2003 and the current coronavirus disease 2019 (COVID-19) pneumonia and the history of the effective responses to epidemics in the past 2,000 years have fully demonstrated the effectiveness of CM in treating infectious diseases. This article discusses the different treatment mechanisms for infectious diseases in $\mathrm{CM}$ and modern medicine and the advantages of $\mathrm{CM}$ methods, which will reacquaint the world with $\mathrm{CM}$. The improvement of the diagnosis and treatment system of CM based on scientific concepts and methods and the organic combination of both treatment methods of modern medicine and $\mathrm{CM}$ will provide the best solution for humans to defeat epidemic infectious diseases.

KEYWORDS COVID-19, coronavirus, infectious disease, epidemic, Chinese medicine, SARS-CoV-2
\end{abstract}

Pneumonia resulting from infection with severe acute respiratory syndrome coronavirus 2 (SARSCoV-2) is currently spreading rapidly around the world. The number of people infected and killed is increasing dramatically. During the global spread of the virus, a variety of mutations have been discovered. ${ }^{(1)}$ As usual, people have pinned their hopes of curbing the virus on the development of a vaccine and antiviral drugs. The development of vaccines based on previously isolated virus strains as well as clinical trials of antiviral drugs are currently being conducted, which appears to give hope to the world facing the threat of coronavirus disease 2019 (COVID-19).

However, it is unclear at present how much therapeutic coverage the vaccine developed based on the isolated virus strains has for the virus causing COVID-19 and its various mutants and whether new viral variants will require new vaccine research. Obviously, it is difficult for the speed of vaccine development to keep pace with the rate of viral mutations. Furthermore, it is also unclear at present whether the antiviral drugs in clinical trials will have the expected effects on severe infections and new variants of SARS-CoV-2 and whether there are unacceptable side effects on the human body. All these uncertainties cast a shadow on the hopes of defeating the virus with new vaccines and antiviral drugs.

During the SARS epidemic in 2003, the excellent results of treatment with Chinese medicine (CM) left a deep impression on the world, and the severe side effects and sequelae caused by the use of interferon, Oseltamivir Phosphate Capsules (Duffy), and high-dose hormones in the treatment of SARS and subsequent influenza A were also memorable. In the face of the COVID-19 epidemic, modern medicine offers only supportive therapy and symptomatic treatments, and there is no medicine to eliminate this viral infection. As a result, $\mathrm{CM}$ has become the mainstay of treatment in China.

From January to April 2020, CM played an important role in the fight against the epidemic in various parts of China, whether in the initial treatment of SARSCoV-2 infection or by saving the lives critical patients, and the effectiveness rate of treatment with CM mostly reached $90 \%$ or greater. ${ }^{(2-10)}$ In the face of COVID-19

CThe Chinese Journal of Integrated Traditional and Western Medicine Press and Springer-Verlag GmbH Germany, part of Springer Nature 2020

Hong Kong Modern Chinese Medicine Research \& Development Center, Hong Kong SAR, China

E-mail: greenisland@ vip.163.com

DOI: https://doi.org/10.1007/s11655-020-3324-z 
pneumonia, which is not cured by modern medicine, why does $\mathrm{CM}$, which has little to do with modern science and technology, have surprising clinical effects?

A Complete System Abandoned in Scientific Garbage: CM Syndrome Differentiation and Treatment System for Epidemic Infectious Diseases

In fact, whether it is SARS, influenza A, or the current COVID-19 pneumonia, similar epidemics have occurred many times in China since ancient times. There are 3 systems for the treatment of epidemic infectious diseases in CM: six channels syndrome differentiation, Wei-Qi-Ying-Xue syndrome differentiation, and Tri-jiao syndrome differentiation. They have been gradually developed by ancient $\mathrm{CM}$ doctors at the cost of countless lives in the long experience of fighting all kinds of epidemic infectious diseases.

ZHANG Zhong-jing was a famous medical scientist who lived during the late Eastern Han Dynasty approximately 1,800 years ago. In less than 10 years after the first year of the Jian'an era, two-thirds of his clan's 200 people died, $70 \%$ died of epidemics. Feeling his powerlessness in the face of the epidemic that killed countless people, ZHANG Zhong-jing began to diligently study ancient medical knowledge, and he extensively collected treatment methods. His handeddown work Treatise on Febrile Disease Caused by Cold (Shang Han Lun) laid the foundation for CM to treat epidemic infectious diseases.

In the late Ming and early Qing dynasties, wars continued, and epidemics spread. According to the "History of the Ming Dynasty", there were 19 major outbreaks from the 6th year of the Yongle Emperor (1408) to the 16th year of the Chongzhen Emperor (1643). In approximately 1,642 severe epidemics occurred in the area south of the Yangtze River for several consecutive years. There were many places where nearly everyone in a family, on a street, or in an entire village died from epidemics. The method described in Treatise on Febrile Disease Caused by Cold was not ideal for the treatment of the epidemics of the time. Therefore, YE Tian-shi, WU Ju-tong, XUE Sheng-bai, WANG Mengying and other clinical doctors successively established the "differentiation of Wei-Qi-Ying-Xue" and the "differentiation of Tri-jiao" based on their clinical practice, which improved the CM syndrome differentiation and treatment system for epidemic infectious diseases of the warm-heat or damp-heat type.

In modern times in China, Western medicine (WM) has gradually flourished and replaced CM to achieve the status of mainstream medicine. CM has been gradually excluded from the treatment of epidemic infectious diseases. Under the banner of science, epidemic infectious diseases described by scientific concepts such as bacteria, viruses and infections have become unfamiliar to the Chinese people who have fought them for nearly 2,000 years, as if similar diseases have never occurred before. In the face of the threat of epidemic infectious diseases, people now think of the concepts of bacteria, viruses, antibiotics, and vaccines and forget that there was a complete system to deal with epidemic infectious diseases within CM under simple concepts such as wind, cold, heat and damp. This system included prevention, initial treatment, treatment for complications, rescue of critically ill patients, and recovery during rehabilitation. Regardless of what type of virus invades and no matter how the virus mutates, from the perspective of $\mathrm{CM}$, they remain one of several types: cold, hot, damp, or a composite type. The possible pathological states of patients at various stages of the development of infectious diseases are described in detail in $\mathrm{CM}$, including the identification of various states, coping methods, and even $\mathrm{CM}$ prescriptions. These were summarized by $\mathrm{CM}$ doctors in the long-term fight against infectious diseases during an era when WM had not yet been introduced into China. The large number of patients cured by this method is recorded in the historical documents of $\mathrm{CM}$. However, with the rise of modern medicine based on modern science, they have been regarded as nonscientific "garbage" and discarded. ${ }^{(11)}$

It should be mentioned that each time an epidemic occurs, from ancient times to the present, the virus that causes it could be more or less different, but the patients' response states and the disease evolution would not vary completely along with the pathogen, which have a certain relationship with the internal structure and function of the human body. Individuals may have different response states to the same invading pathogen, and the response states caused by different invading pathogens may be the same. CM treatment is based on the body's response state. Therefore, from the perspective of treatment, it is not necessary to consider whether the current pathogen is different from ancient ones. Importantly, there is no obvious difference in the response state of the body to a 
pathogen today and in ancient times.

Treatment of Body's Response State: Advantage of CM in Treating Epidemic Infectious Diseases

In modern medicine, research on drugs or vaccines against viruses generally targets drugs that can kill the virus in vitro, with subsequent animal experiments and clinical trials demonstrating that their level of safety is acceptable for humans. Regardless of whether it is an in vitro experiment, an animal experiment or a clinical trial, the reference system for the evaluation of efficacy is the virus. A sign of drug effectiveness is usually a detectable decrease in the quantity or activity of the virus or the complete elimination of the virus.

Studies have confirmed that there are "healthy carriers" who have been documented to carry the virus but not become sick. ${ }^{(12)}$ Studies have also shown that in some patients who have recovered, the virus can still be detected, ${ }^{(13)}$ though such a virus is typically no longer able to harm the carrier. The reason may be that the patient has developed immunity to the virus or that the virus has been inactivated.

Overall, a human infected with the virus may not necessarily become ill, and the disease may also be cured while the virus is still in the body. In other words, the role of the virus itself in the development of viral infectious diseases is not always decisive. Killing the virus can be the direct effect of vaccines or antiviral drugs, or it can be achieved indirectly by mobilizing and strengthening the body's immune system. On the other hand, virological research shows that the damage done by viral infectious diseases to the human body is not always directly caused by the virus. Secondary damage may occur along the inherent causal relationship chain of the disease, which is caused by direct damage due to the virus and the abnormal response state of the human body. Therefore, in the course of a viral infectious disease, even if the virus has been killed, this does not mean that all the problems associated with disease have been solved.

Obviously, treating viral infectious diseases can be achieved directly by action on the virus, indirectly by action on the virus through strengthening of the body's immunity, or by blocking the disease development process through other links in the causal chain of the disease. Of course, treatment can also be achieved by influencing multiple links at the same time to exert synergy. In other words, killing viruses directly is not the only way to treat viral infectious diseases. Focusing on the human response state caused by the virus or on blocking the causal chain of disease development is also likely to achieve the goal of curing viral infectious diseases. ${ }^{(14)}$ The effect mechanisms of some effective drugs that are found by using the body's response state as a reference system may occur by mobilizing the immune system, strengthening the ability of the immune system, or blocking the developmental process of the disease.

Generally, the response state of the body caused by the virus is not determined unilaterally by the virus but is also related to the structure and function of the body part affected by the virus. In other words, in the same part of the body, there are not as many response states as there are kinds of invading viruses, and similar response states may be caused by many different viral infections. For example, COVID-19 pneumonia today, SARS in 2003, and influenza A in 2009 produced the same or similar response states. Clinical manifestations such as fever, cough, headache, and sore throat are all common in the early stages of these different viral infections. Even the early clinical manifestations of upper respiratory infections caused by bacteria are similar.

The structural and functional damage caused by the virus and the body's response to the virus will be reflected in the form of symptoms, signs, abnormal indicators in the analysis of blood and excretions, or abnormal features on omputerized tomography (CT) or ultrasound. After infection with SARS-CoV-2, coughing, expectoration, shortness of breath, and respiratory distress are clear symptoms of lung infection. If the patient has respiratory distress, "ground glass opacities" and "white lung", which are indicative of lung injury, may appear on CT scans, as mentioned above. Vomiting and diarrhea are usually the normal response of the digestive system to viral invasion, but severe vomiting and diarrhea can also lead to lower blood pressure, hyponatremia and hypokalemia. The elevation of liver enzymes, lactate dehydrogenase, muscle enzymes, and myoglobin are indicators of damage to the liver, kidney, and myocardium. Fever is the body's conventional response to viral invasion. Routine blood analysis usually shows that the total number of white blood cells does not increase and the lymphocyte count decreases. Weakness reflects the damage caused by the virus to the human body and the large quantities of physical 
energy consumed in the fight against it. ${ }^{(15)}$

Before modern medicine was introduced into China, given the state of development of science and technology 100 years ago, virus, the pathological changes in internal organs caused by the virus or changes in certain microscopic components of blood and feces could not be seen. However, ancient CM practitioners believed the symptoms and signs during the disease process to be a reflection of the damage to the organs and tissues of the body. The appearance of cough, expectoration, shortness of breath, and respiratory distress meant impaired structure and function of the lungs and respiratory tract. The improvement and disappearance of these symptoms and signs meant that the corresponding internal damage had been repaired. The disappearance of symptoms such as vomiting and diarrhea meant that the damage to the digestive system by the virus had been controlled and that the body's digestive, absorptive and excretory functions had returned.

The 4 response states shown on the right side of Figure 1 represent the most common types of initial body response states in infectious diseases summarized by ancient CM doctors. The efficacy of CMs against infectious diseases was evaluated using these response states as a reference system. Elimination of the symptoms and signs corresponding to a certain response state by a kind of $\mathrm{CM}$ or a mixture based on a CM prescription indicates that the damage to the internal organ structure and function corresponding to this response state has been repaired.

When the focus of the treatment of viral infectious diseases is placed on the response state of the body caused by the virus, it is not necessary to be concerned with which virus is causing the disease, with the continuous mutation process of the virus, or even with whether the disease is caused by a virus or by other pathogens. As long as we find a method to treat this response state and correct it in time and to block disease transmission along a fixed chain of causality, the goal of curing the disease can be achieved. Whether the pathogen is killed or inactivated by the method used to eliminate the pathological response state is not important for curing the disease. In short, the virus loses its pathogenic effect on the body because if it continues its action, the state of the body's abnormal response cannot be corrected, and the damage cannot be repaired. In the

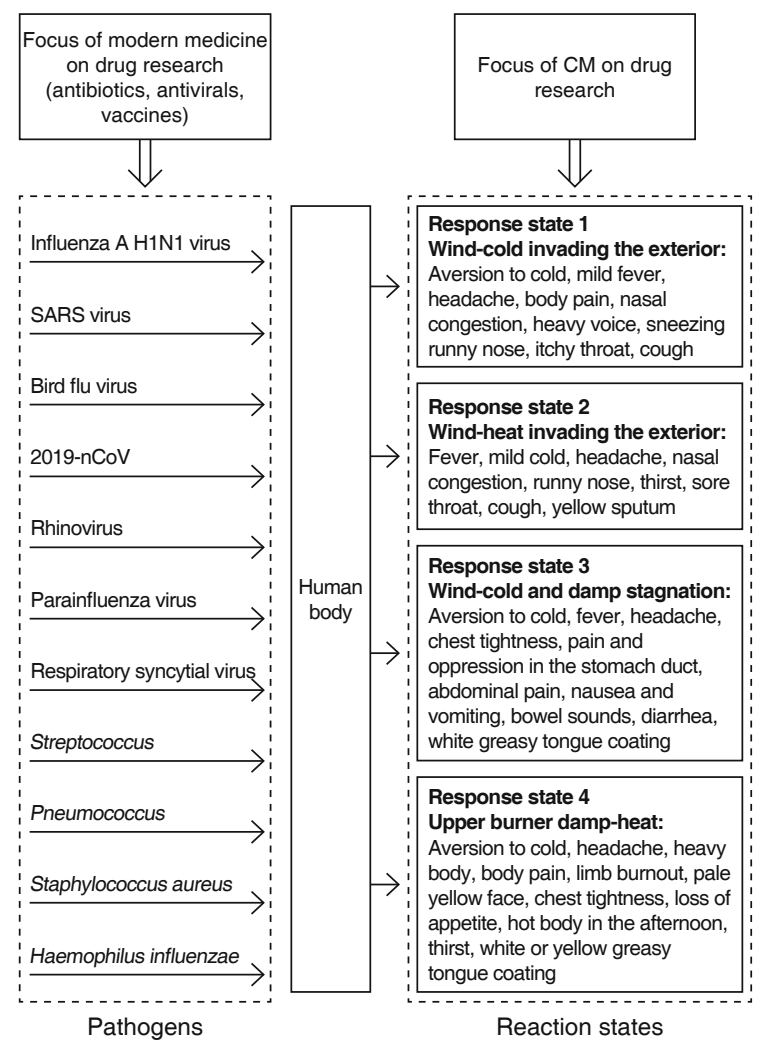

Figure 1. State of Human Body's Response to Pathogens

face of epidemics, research on the treatment methods directed against viruses has always been limited by time and technology and cannot keep up with the pace of the outbreak. Why must we focus on killing the virus instead of seeking solutions targeting other links in the causal chain of the development of viral infectious diseases?

Today, advances in immunological research have revealed the lethality of the human immune system on viruses. This provides another way to kill the virus, i.e., by strengthening the body's immunity, such that the immune system that is damaged in fighting the virus can be continuously supported and supplemented, which may be a more effective way to kill the virus. As shown in Figure 1, effective drugs identified with pathogen killing as the reference criterion for evaluating efficacy, such as vaccines or antiviral drugs, typically directly kill (or inactivate) pathogens in the body but cannot be expected to simultaneously repair the associated damage. After taking the medicine, if repair of the damage occurs, this is usually a function of the body's self-healing ability after the effect of the pathogen has been weakened or eliminated. In contrast, effective drugs identified by using the body's response state as a reference system are definitely able to improve the body's abnormal response 
states caused by pathogens. Their effect mechanism may be direct repairing of the damage caused by the pathogen, mobilizing or strengthening the body's immunity, or acting directly on pathogens; more than one link may also be affected at the same time. In short, regardless of which link in the causal chain is targeted, a drug could be found to be effective as long as it can ultimately improve the body's response state. Obviously, by replacing the reference system for drug research with the body's response state instead of the presence of the pathogen, drug screening will have a broader field of vision, greatly increasing the probability of discovering drugs with a mechanism of action that does not involve in directly killing the pathogen.

Since ancient times, CM's understanding of the causes of epidemic infectious diseases has never been related to real pathogens. The etiological theory established by means of the differentiation of syndromes is to place the understanding of the cause on the body's response state (syndrome). This is why in ancient times, when science and technology were rudimentary, $\mathrm{CM}$ doctors could still find effective ways to deal with large-scale epidemics caused by pathogens similar to coronaviruses.

The treatment method targeting the body response state not only is superior to the method of directly targeting pathogens (such as vaccines) on the broad spectrum of applicable pathogen types, but also repairs the damage caused by the pathogens, so its therapeutic effect is usually comprehensive in multiple aspects. Furthermore, the treatment methods (such as CM compound prescriptions based on syndrome differentiation) targeting the body's response state have been used in $\mathrm{CM}$ for thousands of years, and there is extensive "real-world evidence (RWE)" that these methods can kill (or inactivate) pathogens in the body. Compared with drugs developed through in vitro and animal tests, they typically have greater adaptability and fewer side effects in humans. Clearly, unlike antiviral therapy and immunotherapy directed against pathogens, the method of targeting the body's response state is a safer, more effective and more widely applicable method for dealing with infectious diseases.

Changes in Modern Medicine: toward A New Medical System That Is Compatible with CM Differentiation and Treatment

For nearly a century since modern medicine assumed a mainstream medical position, CM has been excluded from the treatment of infectious diseases. Based on experience from participation in the fight against COVID-19 pneumonia, the current understanding of the $\mathrm{CM}$ community of the occurrence, development and transmission of COVID-19 has become clearer, and a consensus has basically been reached with regard to the treatment options for the initial and intermediate stages. There is also good momentum in the integration of $\mathrm{CM}$ and WM for the treatment of critically ill patients. As introduced by the Academician ZHANG Bo-li, CM syndrome differentiation and treatment combined with respiratory support therapies from WM, such as oxygen inhalation and upper respiratory ventilation, have shown very good results in "reducing lung tissue exudation, alleviating asthma and other symptoms, stabilizing blood oxygen saturation, and protecting important organ functions". (2)

In recent decades, the rescue of critically ill patients has been dominated by modern medicine, and $\mathrm{CM}$ has been largely excluded. Nonetheless, for some difficult problems with modern medicine in this field, the treatment methods of $\mathrm{CM}$ have had good curative effects, such as in treating gastrointestinal insufficiency after shock, adverse reactions to antibiotics for severe infections, secondary infections and drug resistance. Critical patients often exhibit multiple organ dysfunction, such as severe infections, shock, combined renal insufficiency, gastrointestinal insufficiency, and coagulation dysfunction. ${ }^{(16)}$ The formulation of a treatment plan must consider the overall condition of the patient and the interrelationships of all aspects of the disease, which is precisely the advantage of CM. Obviously, taking advantage of $\mathrm{CM}$ to treat critically ill patients and achieve better synergy with rescue and supportive therapies of WM will further reduce the mortality due to viral infectious diseases. In addition, once vaccines or antiviral drugs are successfully developed and put into use, combining them with $\mathrm{CM}$ treatment methods based on the body's response state will comprise a "joint attack" against viral infectious diseases.

According to an article published in Science on January 27th, remdesivir has anti-SARS-CoV-19 activity, and the authors suggested that the combination of remdesivir and monoclonal antibodies may be an ideal treatment for the new coronavirus pneumonia. Why would scientists make this assertion without 
sufficient clinical evidence to support it? In the words of virologist Dr. Mark Denison, "remdesivir is active against every coronavirus we have tested, and I would be surprised if it is not active against COVID-19." ${ }^{(17)}$

Today, the initial response status of COVID-19 pneumonia and the pathological status of the various stages in the development of the disease are not beyond the scope of CM treatment of epidemic and infectious diseases. If there is no response in the patients who have the corresponding syndrome and use the corresponding prescription, it will also surprise CM doctors. Today, an increasing number of clinical reports on the use of CM to treat COVID-19 pneumonia have fully confirmed its effectiveness. ${ }^{(18-23)}$

To date, more than 1.5 million disease-causing viruses have been found, and these viruses often mutate. In general, vaccines are only effective against specific viruses or specific variants of a virus. Although there is also the possibility that a vaccine or antiviral drug is suitable for multiple viruses of one type, the number of vaccines and antiviral drugs that humans need to develop to address various infectious diseases caused by viruses is still enormous. Because the speed at which vaccines and antiviral drugs are introduced cannot keep pace with the speed of viral mutations, the investment of enormous amounts of human and material resources for drug development is likely to resemble using a "bamboo basket to draw water". In addition, there are other potential issues: years may be required for the regulatory approval of vaccines or antiviral drugs, and even if a drug is approved, it may have side effects. During the swine flu epidemic from 2009 to 2010, 6 million people received the Pandemrix vaccine produced by GlaxoSmithKline. However, the vaccine was recalled after it was discovered that it could cause narcolepsy in some people. ${ }^{(24)}$

The research and development of a single vaccine is already difficult. Faced with 1.5 million types of viruses that are constantly mutating, the oneto-one development of vaccines and antiviral drugs against a virus is obviously not the ideal solution. Academician ZHANG Xue-min, who revealed the important regulatory mechanism of the cell "door god" against viral infection, said that scientists hope to find a "public solution" to viral infection from a perspective of commonality. "Find a fixed method that allows us to deal with the attack of any virus", ${ }^{(25)}$ and the CM treatment method focusing on the response state of the body to viruses is exactly this method.

Overall, the premise of treatment based on the body's response state is as follows: as long as the body's response state appears the same, the same treatment method can be used, even though the invading virus is different. The current description of $\mathrm{CM}$ on the human body's possible states in various diseases is achieved through a small number of syndromes (approximately 100 types), no more than 50 of which are related to infectious diseases caused by pathogens. The syndromes are equivalent to the state variables used by modern science to describe the state of a system. It is not necessary to consider 100 state variables; even if there are only 10 , the number of personalized states that can be described by combinations is also an astronomical figure. ${ }^{(26)}$ Clearly, this is a system capable of "controlling complex things using a simple method". Throughout the long process of fighting against all kinds of infectious diseases, ancient $\mathrm{CM}$ doctors have basically understood the types of response states that occur after various pathogens invade the human body and the change law of the body's response states during the evolution of a disease. For each syndrome, there are effective treatment methods and prescriptions. Therefore, for every epidemic outbreak, a well-trained CM physician can rapidly determine the type of response state in the early stages, the evolutionary law of the disease, and feasible treatment methods based on the diagnosis and treatment system passed down by ancients.

$\mathrm{CM}$ is a personalized medical system. With the rise of precision medicine in recent years, modern medicine has begun to explore drug evaluation systems suitable for personalized medicine, such as "basket trials" and "umbrella trials". ${ }^{(27)}$ In 2017, the U.S. Food and Drug Administration (FDA) for the first time approved indications and drugs that do not target diseases but target biomarkers that can appear in a variety of diseases. ${ }^{(28)}$ In this context, the evaluation of the efficacy of CM should naturally go beyond the limitations of taking the modern medical disease classification system as the sole frame of reference, and CM (or compound $\mathrm{CM}$ ) is no longer required to be applicable to different populations and different types under one disease. The "basket trial" model is suitable for evaluating drugs applicable to the same response state caused by different pathogen infections, and the "umbrella 
trial" model is suitable for evaluating multiple drugs applicable to multiple different response states caused by the same pathogen infection. The goal of launching precision medicine in the United States is to establish a new disease classification system that is different from the traditional disease classification system. ${ }^{(29)}$ The integration of CM syndromes into modern medicine is equivalent to the establishment of another new disease classification system under the framework of modern medicine. On the surface, CM's description language based on qi, blood, yin and yang and that of modern medicine based on biology, physiology, biochemistry, and cell and molecular biology appear to be totally unrelated. Today, the idea of complexity science has gradually become the mainstream scientific idea. People have applied the "sand pile" model to reveal the formation of earthquakes, forest fires, biological extinctions and even urban traffic jams, and the laws revealed by the "butterfly effect" have been widely used in various dynamic systems. Therefore, why can we not use models that include the concepts of qi, blood, yin and yang to reveal the physiological and pathological laws of real human existence?

Today, large-scale clinical trials featuring randomized double-blind controlled trials remain the gold standard for testing the safety and effectiveness of treatment methods. However, in the past, drugs developed based on this seemingly rigorous scientific method have often been found to have some long-term side effects after their clinical application for a period of time, which in severe situations have even led to the elimination or ban of the drug. An increasing number of people are beginning to question the reliability of the results of such clinical trials, and the economics and rationality of such clinical trials are also being increasingly criticized. In this context, RWE is receiving increasing attention. Currently, the U.S. FDA has begun to introduce RWE into the evaluation of the reliability of newly approved drugs in real populations. ${ }^{(30,31)}$ Among populations with infectious diseases, many people have more than one CM syndrome, i.e., the response state caused by the pathogen is reflected as a combination of different syndromes, and this combination manifests as personalized characteristics in the population. These factors make it more difficult to evaluate the efficacy of treatments using randomized controlled trials. Therefore, when dealing with COVID-19 pneumonia, how can a treatment plan be formulated based on CM's previous experience gained from the real world? In the actual diagnosis and treatment process, how can the efficacy of $\mathrm{CM}$ be evaluated based on data from real-world research? It is a challenge for humans to overcome epidemic infectious diseases with the help of $\mathrm{CM}$. At the same time, it is also an excellent opportunity for the world to understand the scientific and practical value of $\mathrm{CM}$.

\section{Conclusions}

In the past 2,000 years of fighting against infectious diseases, Chinese ancestors created a comprehensive response system including diagnosis and treatment at the cost of countless lives, which has guaranteed the survival and prosperity of the Chinese nation. Currently, in the face of the ravages of coronaviruses, $\mathrm{CM}$ doctors are at the forefront of fighting the epidemic. In the struggle against this unprecedented virus, these doctors have achieved amazing curative effects through the methods and techniques passed down from their ancestors, showing the world the "magic" of CM.

In today's natural sciences, the understanding of the world's origins based on theoretical physics is increasingly moving toward ideas that are consistent with Eastern philosophy. In the life sciences, the holistic concept of systems biology and the individualized concept of precision medicine are driving the return of life sciences to $\mathrm{CM}$ at the methodological level. Drawing on the experience of applying Artemisia annua to treat malaria in $\mathrm{CM}$, modern medicine has developed artemisinin. Based on acupuncture and moxibustion, which were applied for thousands of years in CM, trigger point acupuncture (dry needling) therapy has emerged in modern medicine. Today, the reform of the clinical evaluation system brought about by the rise of precision medicine has opened the door for incorporating the diagnosis and treatment system of CM based on the human response state into modern medicine. Incorporating the personalized medical system of $\mathrm{CM}$ into modern medicine and then using modern scientific norms and technical means to scientize CM will mean that modern medicine has truly abandoned the reductionist approach with decomposition and analysis at its core and is beginning to move toward a new medical system that can integrate Eastern medicine and WM and focus on an overall synthesis. The organic combination of the two methods of $\mathrm{CM}$ and WM for treating viral infectious diseases will undoubtedly provide the best solution to overcome epidemic infectious diseases. 


\section{Conflict of Interest}

The author had no conflict of interest to declare.

\section{REFERENCES}

1. Breaking news. Researchers from multiple countries claim that the new coronavirus has mutated. March 5, 2020. Available at https:// news.rthk.hk/rthk/ch/component/k2/1512595-20200305.htm

2. Reporters Tian $\mathbf{X H}$ and Wen $\mathrm{JH}$. TCM can play a full role in the prevention and treatment of COVID-19 pneumonitis-Interview with Academician ZHANG Bo-li, expert of National Scientific Research Expert Group. Xinhua News Agency, Beijing, Jan 27, 2020. Available at http://www.xinhuanet.com/2020-01/27/c_1125505871.htm

3. Liang $\mathrm{Q}$. Integrated traditional Chinese and Western medicine for COVID-19 pneumonia. Xinhuanet, Feb 12, 2020. Available at http:// www.xinhuanet.com/politics/2020-02/12/c_1125561735.htm

4. Huang B, Chen LJ, Suo XC. Let all diagnosed patients use TCM. China Traditional Chinese Medicine Network, Feb 14, 2020. Available at http://www.cntcm.com.cn/2020-02/14/content_71210.htm

5. Wu Q. 29 patients with COVID-19 pneumonia in Shanxi were cured and discharged after integrated Chinese and Western medicine treatment. China News Network, Feb 12, 2020. Available at https:// m.chinanews.com/wap/detail/zw/sh/2020/02-12/9089028.shtml

6. Guangdong Provincial Drug Administration. The Provincial Drug Administration intends to include the registration of preparations for medical institutions in the No. 8 People's Hospital of Guangzhou "Pneumonia No.1" into the emergency approval. Feb 1, 2020. Available at http://mpa.gd.gov.cn/gkmlpt/content/2/2881/post_2881418.html

7. Reporter Guo XY and Zhou Y. Guangdong: The designated hospitals in the province have used "Pneumonia No. 1" over 6,000 packets. Feb 9, 2020. Available at http://news.cnr.cn/native/ city/20200219/t20200219_524982385.shtml

8. Reporter Yi YL. Hunan does have a set, in fighting against plague with TCM. Tencent, Feb 13, 2020. Available at https://new.qq.com/ rain/a/20200213AOMTYO

9. Beijing Municipal Government Information Office. Beijing Ditan Hospital: $87.5 \%$ effective rate of traditional Chinese medicine plus symptomatic treatment, $92.3 \%$ effective rate of integrated traditional Chinese and Western medicine. Tencent, Feb 18, 2020. Available at https://new.qq.com/rain/a/20200215A0NQ3K00

10. Reporter Ma FC. TCM rescue and treatment of patients with COVID-19 pneumonia is promising. China Youth Daily, Mar 3, 2020. Available at http://www.xinhuanet.com/2020-03/03/c_1125657175.htm

11. Yuan B. Dawn of viral epidemic treatment: the medical system based on body states control. Ann Clin Lab Res 2016;4:3.

12. Chang D, Xu HW, Rebaza A, Sharma L, Cruz CSD. Protecting health-care workers from subclinical coronavirus infection. Lancet Respir Med 2020;8:e13.

13. $\mathrm{Yu}$ JM, Zheng HZ. 13 patients who were cured and discharged from hospital turned positive in nucleic acid and experts: whether it is contagious or not is uncertain. Southern Daily, Feb 25, 2020. Available at https://xw.qq.com/cmsid/20200225A0NG5B00

14. Yuan B. Ebola strikes, it is both a challenge and an opportunity. China Traditional Chinese Medicine News, Oct 20, 2014. 3rd Edition, Perspective.

15. General Office of the National Health Commission, Office of the
State Administration of TCM. Notice on printing and distributing pneumonia diagnosis and treatment plan for new coronavirus infection (trial version 6), Feb 5, 2020. Available at http://yzs.satcm.gov.cn/ zhengcewenjian/2020-02-19/13221.html

16. Liu QQ. First aid mainly depends on Western medicine? Wrong! Chinese medicine first aid is not optional, but essential! Daily Headlines, Mar 16, 2020. Available at https://kknews.cc/health/284ynxy.html

17. Cohen J. Can an anti-HIV combination or other existing drugs outwit the new coronavirus? Science, 2020. Available at https:// doi:10.1126/science.abb0659

18. Qing GC, Zhang H, Bai Y, Luo Y. Traditional Chinese and Western medicines jointly beat COVID-19 pandemic. Chin J Integr Med 2020;26:403-404.

19. Yang $Q$, Sun $Q G$, Jiang $B, X u$ HJ, Luo M, Xie $P$, et al. Retrospective clinical study on treatment of COVID-2019 patients with integrated traditional Chinese and Western medicine. Chin Tradit Herb Drugs (Chin) 2020:1-5. Available at http://kns.cnki.net/kcms/detail/12.1108. R.20200413.1302.002.html

20. Song $\mathrm{P}$, Zhao LH, Li XY, Su JS, Jiang ZY, Song B, et al. Interpretation of TCM part in diagnosis and treatment protocol for COVID-19 (trial version 7). J Tradit Chin Med 2020:1-41. Available at http://kns.cnki. net/kcms/detail/11.2167.R.20200325.1623.002.html

21. Xia WG, An CQ, Zheng CJ, Zhang JX, Huang M, Wang Y, et al. Clinical observation on 34 patients with novel coronavirus pneumonia (COVID-19) treated with integrated traditional Chinese and Western medicine. J Tradit Chin Med 2020;61:375-382.

22. Fu XX, Lin LP, Tan XH. Clinical study on 37 case of COVID-19 treated with integrated traditional Chinese and Western medicine. Tradit Chin Drug Res Clin Pharmacol (Chin) 2020:1-9. Available at http://kns.cnki.net/kcms/detail/44.1308.R.20200319.1644.002.html

23. Cui HT, Li YT, Guo LY, Liu XG, Wang LS, Jia JW, et al. Traditional Chinese medicine for treatment of coronavirus disease 2019: a review. Tradit Med Res 2020;5:65-73.

24. Harper J. Coronavirus: will someone develop a vaccine? The BBC, Feb 13, 2020. Available at https://www.bbc.com/news/business-51454859

25. Reporter Li C. Scientists reveal important regulatory mechanism of cell "Door God" against viral infection. China News Network, Feb 23, 2019. Available at https://tech.huanqiu.com/article/9CaKrnKilTz

26. Yuan B. Precision medicine: to start the era of status medicine to be the mainstream. Avid Sci, 2016. Available at https://avidscience. com/book/precision-medicine-to-start-the-era-of-status-medicine-tobe-the-mainstream-medicine/

27. Yuan B. Toward holistic medicine and holistic biology: life sciences after precision medicine and systems biology. Front Life Sci 2019;12:14-26.

28. The ASCO Post. FDA approves larotrectinib for solid tumors with NTRK gene fusion. Dec 27, 2018. Available at https://ascopost.com/ News/59498

29. Ashley EA. Towards precision medicine. Nat Rev Genet 2016;17:507-522.

30. American Society of Clinical Oncology. CancerLinQ partners with FDA to study real-world use of newly approved cancer treatments. Jun 1, 2017. Available at https://www.asco.org/about-asco

31. Gabay M. 21st century cures act. Hosp Pharm 2017:52:264-265.

(Accepted July 22, 2020; First Online October 10, 2020) Edited by YU Ming-zhu 